\title{
LA LÉGENDE NOIRE DU SERVICE D'INFORMATION MILITAIRE DE LA RÉPUBLIQUE DANS LA GUERRE CIVILE ESPAGNOLE, ET L'IDÉE DE CONTRÔLE POLITIQUE
} François Godicheau

\author{
La Découverte | «Le Mouvement Social »
}

2002/4 n n $^{\circ} 201$ | pages 29 à 52

ISSN 0027-2671

\section{Article disponible en ligne à l'adresse :}

http://www.cairn.info/revue-le-mouvement-social-2002-4-page-29.htm

\section{Pour citer cet article :}

François Godicheau, « La légende noire du Service d'Information Militaire de la République dans la guerre civile espagnole, et l'idée de contrôle politique », Le Mouvement Social 2002/4 (n $\left.{ }^{\circ} 201\right)$, p. 29-52.

DOI 10.3917/lms.201.0029

Distribution électronique Cairn.info pour La Découverte.

(C) La Découverte. Tous droits réservés pour tous pays.

La reproduction ou représentation de cet article, notamment par photocopie, n'est autorisée que dans les limites des conditions générales d'utilisation du site ou, le cas échéant, des conditions générales de la licence souscrite par votre établissement. Toute autre reproduction ou représentation, en tout ou partie, sous quelque forme et de quelque manière que ce soit, est interdite sauf accord préalable et écrit de l'éditeur, en dehors des cas prévus par la législation en vigueur en France. Il est précisé que son stockage dans une base de données est également interdit. 


\section{La légende noire du Service d'Information Militaire de la République dans la guerre civile espagnole, et l'idée de contrôle politique}

\section{par François GODICHEAU*}

$\mathbf{L}$

a guerre civile espagnole apparait souvent comme un drame où s'affrontent des acteurs politiques clairement identifiés, pourvus de projets politiques bien délimités.

Dans le camp républicain, la complexité et l'hétérogénéité des organisations de gauche ou de Front populaire ont longtemps disparu sous un étiquetage politique fait de multiples "-ismes", dans une historiographie où le récit de souvenirs et l'analyse critique ont souvent eu partie liée.

A l'intérieur du camp antifranquiste, chaque organisation politique ou syndicale, chaque sous-groupe ou personnalité importante a tenu à donner sa propre version des faits, sa justification ou son explication de la défaite. Aussi longtemps que la dictature franquiste a duré, ces écrits sont restés, avec la presse, les seuls matériaux dont pouvaient disposer les historiens. Aujourd'hui, ils constituent encore une matière sans doute indispensable à qui veut entreprendre l'étude approfondie de la révolution et de la guerre d'Espagne, mais aussi une matière dangereuse car elle propose des éléments d'explication, des pièces d'un puzzle que l'on retrouve quelquefois intactes et fausses dans les meilleurs récits historiques.

Dans son livre récent sur les Brigades Internationales, Rémi Skoutelsky a relevé l'une de ces erreurs qui perdurent de livre en livre, un de ces faux qui deviennent vrais par le simple charme de la répétition : il s'agit de la fameuse réunion du Comintern qui aurait, le 25 juillet 1936, décidé de la création des brigades (1). Carlos Serrano avait déjà montré que les premiers à parler de cette réunion avaient été les propagandistes de la cause franquiste à l'étranger, en France et en Angleterre notam-

* Membre de la Casa de Velázquez (École des Hautes Études Hispaniques).

(1) R. Skoutelsky, L'espoir guidait leurs pas. Les volontaires français en Espagne républicaine, Paris, Grasset, 1998. Préface d'A. Prost.

Le Mouvement Social, n 201, octobre-décembre 2002, @ Les Éditions de l'Atelier/Éditions Ouvrières 
ment (2). Répétant à l'envie l'histoire de cette réunion, ils en avaient fait une référence que l'on retrouvait depuis dans tous les livres. Une fois créée, cette dynamique ne pouvait que se renforcer au fil des ans : cette date était vraie puisqu'elle se trouvait dans tous les livres. Rémi Skoutelsky, le premier à avoir eu accès à la documentation originale du Comintern et des brigades, a montré que ce jour-là, il n'y avait simplement pas eu de réunion au Comintern pour décider de l'envoi d'internationaux en Espagne et que la décision était plus tardive et prise dans d'autres circonstances.

On pourrait faire le même type d'observation dans le cas du Servicio de Información Militar (S.I.M.) et de sa "légende noire". Deux logiques président à la naissance de celle-ci. La première est celle de la caractérisation politique familière à la pratique de l'époque: une personne ou une entité est caractérisée politiquement quand elle reçoit un identifiant souvent définitif, qui la ramène et la réduit à une attitude ou à une "ligne" politique. La seconde est celle du complot communiste, due au climat de guerre froide dans lequel ont été rédigés les premiers récits historiques. Le S.I.M., organisme de contre-espionnage et de police militaire, est devenu l'exemple même de la "toute puissance" du N.K.V.D. (3) en Espagne républicaine et de son travail de répression des opposants politiques, cela jusque dans des publications récentes (4).

Cet article est conçu comme une contribution à la réflexion méthodologique sur l'histoire des relations entre partis et institutions. Il cherche à s'éloigner des thématiques de la manipulation et du complot, souvent présentes quand l'histoire politique fonctionne à partir d'un jeu d'étiquettes politiques. Dans ce jeu trop simple, un individu, une institution ou un groupe, quand ils sont affublés de tel ou tel identifiant politique par l'historien, le sont une fois pour toutes et sont alors censés agir d'une façon déterminée. Certains objets historiques risquent à ce régime de voir leur complexité recouverte d'une toile opaque, une fois rapportés à d'autres "phénomènes" appelés communisme, libéralisme, anarchisme, devenus autant d'évidences, une fois caractérisés politiquement. C'est le cas du S.I.M.

L'objet de l'étude ne saurait être seulement l'organisme S.I.M. Il serait très difficile d'aller plus loin que la description de son organisation interne et de ses fonctions, que nous proposons cependant ici pour la première fois : les archives de cet organisme de contre-espionnage ont en effet été systématiquement détruites devant l'avancée des troupes ennemies. Mais c'est précisément cette caractéristique qui fait l'intérêt du S.I.M. comme objet historique : il est avant tout une légende, une légende noire, et, faute de documentation pour l'étudier, il l'est resté jusqu'à nos jours, cas singulier dans l'histoire de la guerre civile espagnole. Pendant la dictature franquiste,

(2) C. Serrano, L'enjeu espagnol, Paris, Messidor/Éditions Sociales, 1987.

(3) Narodny kommissariat unoutrennik del., commissariat au peuple à l'Intérieur : police secrète soviétique, organisme qui a succédé à l'O.G.P.U., et dont le responsable le plus connu en Espagne était Orlov.

(4) Dans la polémique récente sur la vie politique de Arthur London, le fait qu'il ait travaillé pour le S.I.M. devient une marque d'infamie et suffit à en faire un agent direct de la répression des ennemis de Moscou. Dans Le liure noir du communisme (S. CouRToIs et al., Paris, Robert Laffont, 1997), la contribution de J.-L. PANNÉ reprend sans les examiner les éléments les plus vulgarisés de la légende noire du S.I.M. (p. 365-386). 
de nombreux thèmes de l'histoire du conflit furent l'objet de légendes ou d'hypothèses impossibles à vérifier, mais avec le retour à la démocratie, les historiens ont pu étayer leurs connaissances grâce à l'accès aux archives et proposer un récit plus vraisemblable des événements. Le S.I.M., lui, est resté du domaine de la légende.

Celle-ci a un intérêt en soi. Il ne s'agit pas seulement de l'écarter au profit d'une meilleure connaissance de ce service. Elle est largement symptomatique des défauts qu'a longtemps eu l'histoire politique de la guerre civile et des dangers qui guettent toujours le chercheur dans ce domaine. La légende noire du S.I.M. doit beaucoup aux idées de manipulation et de contrôle politique absolu par un Parti communiste dont on a surévalué les forces et l'homogénéité, elle doit beaucoup au contexte de guerre froide dans lequel elle s'est développée. L'objet en lui-même se prêtait à ces dérives : espionnage, services secrets, cinquième colonne sont autant de thèmes où le mystère se voit souvent expliqué par les a priori de celui qui en fait l'histoire, une histoire souvent "à sensations". Pourtant il nous semble important, même dans l'après-guerre froide, de détailler le fonctionnement de cette légende noire, d'introduire le doute, grâce à une documentation nouvelle et indirecte, sur ses principales assertions, en particulier le contrôle absolu du S.I.M. par le Parti communiste d'Espagne.

\section{Police et contre-espionnage au début de la guerre civile}

Au lendemain du soulèvement franquiste des 18 et 19 juillet 1936 contre la République, celle-ci se retrouve sans armée et ce sont les milices populaires, créées à l'initiative des syndicats et des partis ouvriers et républicains, qui affrontent les rebelles les premières. Au-delà de l'armée, cette situation affecte tout l'État républicain, presque anéanti par le coup d'État militaire et le contrecoup révolutionnaire. Au cours de l'automne 1936, un appareil militaire nouveau se met en place: "l'Armée populaire" est en train de naitre. Tout est à reconstruire, en particulier dans les domaines très sensibles de l'espionnage, du contre-espionnage et de la sécurité militaire : les anciens services de la République étant soit passés à la rebellion soit connus de celle-ci.

Le 17 septembre 1936, sont créées par décret les Milices de Surveillance de l'Arrière qui doivent inclure tous les éléments armés du territoire pour les contrôler. Elles sont censées débusquer les hypothétiques espions et traitres infiltrés dans les milices et ont aussi une mission de simple police à Madrid. Plus tard, sont mises en place les Brigades mixtes et les premières divisions véritablement militaires. Les milices sont militarisées et un commandement unique est institué en mai 1937 avec la création du ministère de la Défense et la restructuration de l'état-major central.

Il existait au sein des diverses instances des milices et de l'armée et dans les états-majors des embryons de services de renseignements - appelés secondes sec- 
tions ; des agents travaillaient à l'étranger pour le gouvernement, mais rien n'était véritablement organisé au sein d'un même appareil efficace et fonctionnant selon des normes fixes avant le mois d'août 1937. Dans le domaine de la police militaire, depuis la fin du mois de septembre 1936 un comité appelé Cabinet d'Information et de Contrôle était chargé de l'épuration du personnel militaire. Un premier service d'espionnage et de contre-espionnage était partagé entre le Département Spécial d'Information de l'État (D.E.D.I.D.E.), organisme créé en mai 1937 à partir des Milices de Surveillance de l'Arrière (avec à la fois une mission de police et de renseignement intérieur) et le Service d'Information Spécial Périphérique (S.I.E.P.), chargé du renseignement en territoire ennemi et à l'étranger.

Le Service d'Information Militaire est créé le 9 août 1937, sur l'initiative du ministre de la Défense, le socialiste Indalecio Prieto. Peu à peu, il absorbe et centralise tous les autres organismes. Seul l'espionnage proprement dit reste aux mains du S.I.E.P. Deux raisons expliquent cette création. La première est l'efficacité d'un service unique placé directement sous les ordres du ministre et soumis à lui seul (il introduit dans le décret une clause qui stipule que toutes les nominations devront revêtir sa signature originale), condition nécessaire pour éviter qu'il ne tombe sous la coupe d'un seul parti, en l'occurrence le Parti communiste d'Espagne. La deuxième raison est le besoin d'en finir avec les abus commis dans les unités militaires par une police militaire improvisée et dont les actions sans contrôle pouvaient nuire à l'image de la République.

C'est dès la création du S.I.M. que naît le soupçon qu'il est un instrument des communistes et en particulier des Russes. Prieto admet que le chef de la délégation des services secrets soviétiques, Orlov, demandait depuis longtemps la création d'un appareil centralisé dont il aurait été l'assesseur technique; il confesse même avoir laissé à Orlov le soin de la rédaction du décret de création du S.I.M., s'attribuant seulement la paternité de la clause sur l'exclusivité des nominations (5). Depuis lors, tout est venu renforcer l'image d'un S.I.M. appendice du N.K.V.D. : l'importance politique et diplomatique des Russes en Espagne, l'ascension extraordinaire des communistes au cours de la guerre et la chute de Prieto au printemps de 1938. Y contribuèrent aussi les accusations des anarchistes et des poumistes qui se considéraient victimes du S.I.M. puisqu'ils avaient été victimes de l'agressivité et de la volonté d'épuration staliniennes; enfin, il faut signaler l'importance de la victoire franquiste et de la large diffusion d'écrits biographiques qu'elle a permise sur la terrible cruauté du "S.I.M. rouge". Tous ces éléments ont forgé une légende noire que jamais aucune étude n'est venue confirmer, infirmer ou simplement préciser.

(5) Prieto Tuero, Convulsiones de España : Cómo y porqué salí del Ministerio de Defensa Nacional : intrigas de los rusos en España, Barcelone, Fundación Indalecio Prieto/Editorial Planeta, 1989. Pour le S.I.M. des Brigades, on lira le chapitre correspondant de R. SkOUTELSKY (op. cit., p. 254 et sq.). Pour un tableau, non dénué d'erreurs, des différents services de sécurité du début à la fin de la guerre, on peut se reporter à un article de S. NUÑEZ DE PRADO y CLAVELL, "Los servicios de información republicanos (1936-1939)", Anuario del departamento de Historia, 1991, p. 31-43. Plus largement, on peut trouver des informations dans les livres de D. PASTOR PETT, La cinquena columna a Catalunya (1936-1939), Barcelone, Galba, 1978, et Los dossiers secretos de la guerra civil, Barcelone, Argos, 1978. 
Faute d'archives du S.I.M., nous nous sommes appuyé sur une source indirecte pour proposer une description de l'organigramme et des activités du S.I.M. de la région de Madrid, nécessaire pour montrer que ce service était avant tout une partie de l'appareil d'État de la République espagnole en guerre dont, d'une certaine manière, il reflète certaines particularités. Cette source, c'est le dossier d'instruction du procès intenté au chef du S.I.M. de Madrid, Angel Pedrero García, et à ses nombreux subordonnés par les juges au service de Franco de 1939 à 1941. Ce procès s'inscrivait dans une entreprise plus vaste qui visait à étayer le récit des méfaits des "rouges" et qui fut appelée la Causa General (6).

La qualité de cette documentation (7) nous permet d'aborder avec un relatif détail la question du "contrôle" politique par le Parti communiste de l'activité du S.I.M. ou de certains de ses agents. Même s'il est impossible de mener cette enquête au-delà de la région centre (Madrid), nous verrons grâce à d'autres documents plus dispersés que l'on peut étendre à tout le territoire républicain les réticences devant la légende noire du S.I.M.

\section{L'histoire du S.I.M. de 1937 à nos jours : la légende d'un monstre politique}

On l'a dit, cette légende prend ses racines dans la guerre elle-même : le S.I.M. apparaissait comme la partie la plus inquiétante d'un système étatique républicain de plus en plus policier et qui était l'objet d'une lutte d'influence féroce entre les différents groupes politiques. Le caractère nécessairement secret de ses activités le prédestinait à recevoir les critiques avant même qu'il ne s'illustre par des mauvais traitements infligés aux prisonniers ou par un esprit partisan quelconque. L'organisation qui détenait les positions les plus fortes au sein de la police et en particulier à

(6) Ce vaste procès de l'Espagne "rouge" était censé avoir des résultats spectaculaires puisque le "Caudillo " Franco parlait d'un million de victimes de la guerre et de la répression sanguinaire menée par l'ennemi. Le résultat fut tellement inférieur au chiffre promis (85940 morts, chiffre encore très exagéré), malgré toute la bonne volonté des juges qui comptaient de nombreuses victimes plusieurs fois, que le livre-résumé de la Causa ne fut publié qu'en 1961: Causa General. La dominación roja en España. Avance de la información instruida por el ministerio público, Madrid, Ministerio de Justicia. Les fonds sont actuellement conservés à l'Archivo Histórico Nacional à Madrid.

(7) Au cours de cette entreprise, les procédés employés pour réunir les preuves étaient de trois sortes : l'enquête par lettres auprès des nouvelles autorités municipales, l'examen des archives de la justice républicaine et dans une moindre mesure l'interrogatoire de témoins et de "victimes des rouges". Le procès Pedrero est le volume où l'on trouve le plus d'interrogatoires de "rouges " eux-mêmes, en l'occurrence des fonctionnaires du S.I.M. Près de 400 d'entre eux furent arrêtés et isolés dans différentes prisons. Leurs témoignages furent systématiquement confrontés entre eux, aux documents du S.I.M. qui avaient échappé à la destruction et à ce que savaient les services de renseignement franquistes sur tel ou tel individu ou affaire. Les accusés ne signaient pas d'aveux délirants; en revanche, ils répondaient aux questions très précises de juges préoccupés par la qualité des preuves et respectueux des procédures, même s'ils étaient marqués de tous les préjugés des vainqueurs de la "croisade". 
la Direction Générale de la Sécurité (D.G.S.) était sans aucun doute le Parti communiste d'Espagne, ce qui ne signifie pas qu'il était tout puissant ni qu'il était le seul parti à avoir des objectifs de pouvoir. Des exemples d'abus de la part de fonctionnaires du S.I.M. (malheureusement insuffisamment documentés) et la lutte personnelle que menait depuis longtemps le ministre de la Justice Manuel de Irujo (nationaliste basque) contre l'influence communiste dans l'appareil répressif de la République (8) expliquent qu'un certain nombre de députés avaient protesté contre les abus du S.I.M., comme le fit Manuel de Irujo lui-même en Conseil des ministres le 10 août 1938 (9).

Les dénonciations les plus vibrantes furent faites après la guerre par des franquistes, anciennes " victimes du S.I.M. ». Plusieurs livres aux titres évocateurs furent publiés dès le lendemain de la victoire nationaliste : Terreur rouge! Les checas de Barcelone. Histoire de la Barbarie marxiste dès 1939 par Agustí de Foxa (10), Images de la captivité chez les Rouges : mémoires d'un prisonnier du S.I.M. à la Librería Religiosa par Miguel Sabater (11). Pourtant, dans ces livres, le S.I.M. n'est pas sérieusement impliqué : Martin Inglés par exemple, auteur de Dans les griffes du S.I.M., les Checas de Barcelone chez le même éditeur (12), accuse d'un certain nombre de mauvais traitements (passages à tabac) un policier de Barcelone qui, vérification faite, n'était pas membre du S.I.M., mais de la Brigade d'Investigation Criminelle qui dépendait de la D.G.S. de Barcelone et fonctionnait en liaison avec les commissariats de police. L'auteur, quand il décrit des supplices plus cruels, ne fait de son propre aveu que reprendre les dires d'un compagnon de captivité rencontré par la suite dans la prison centrale de la ville.

Les mauvais traitements et le contrôle exercé par les Soviétiques et en particulier par le responsable des services spéciaux, Orlov, sont les deux éléments qui ont poursuivi le S.I.M. dans toute l'historiographie de la guerre civile. Un des meilleurs livres sur l'histoire politique du camp antifranquiste, celui de Burnet Bolloten (13),

(8) Un des aspects de cette lutte était l'intérêt pour éclaicir la question de la disparition d'Andrés Nin (enlevé et assassiné par le N.K.V.D.), pour faire respecter le travail de la justice face à l'arbitraire de la police.

(9) Le S.I.M. avait généralement mauvaise réputation car ses méthodes étaient brutales et arbitraires, et ceci d'autant plus que la guerre avançait vers la défaite républicaine. En Catalogne, s'ajoutaient à ces raisons le manque d'égard de nombreux responsables et agents de ce service envers la culture et la spécificité catalanes, sans parler des prérogatives des institutions de cette région. Le dernier responsable du S.I.M., Santiago Garcès, allègue aussi le fait que les services de sécurité et de censure ont rarement bonne réputation.

(10) ¡Terror rojo! Las checas de Barcelona. Historia de la barbarie marxista, Barcelone, Impr. Giménez, 1939, ou encore T. BorRas, Chekas de Madrid. Epopeya de los caídos, Madrid, Editorial Nacional, 1944. Les "checas " était le nom donné dès 1936 dans le territoire républicain aux unités de police mises en place par les partis ouvriers pour remplacer la police et réprimer les franquistes au lendemain du soulèvement, en référence à la Tchéka soviétique. Par extension, le terme désigne les lieux de réclusion utilisés par ces brigades policières. La plupart furent dissoutes pendant l'hiver 1936-1937 et seules un certain nombre de prisons "improvisées " subsistèrent, comme le couvent de Santa Ursula à Valence ou la prison de la rue Puerta del Angel à Barcelone.

(11) Estampas del cautiverio rojo : memorias de un preso del S.I.M., Barcelone, 1940.

(12) Bajo las garras del S.I.M. Las Chekas de Cataluña, Barcelone, 1940.

(13) Madrid, Alianza Editorial, 1993. Ce livre, malheureusement pas édité en France, est le plus com- 
La guerra civil española..., est sans doute le plus bel exemple de la façon dont la légende noire a été véhiculée. On y retrouve des citations de tous ceux qui ont contribué à la forger. La thèse est simple :

Le S.I.M. fut crée sans aucun doute pour couvrir d'un voile de légalité les desseins criminels du Parti communiste. Le caractère secret des activités de contre-espionnage fournissait aux inspirateurs et aux hauts fonctionnaires du S.I.M. la carte blanche idéale, qui leur permettait d'agir en toute impunité (14). [...] Même si les fonctions du S.I.M. étaient de combattre l'espionnage, d'empêcher les actes de sabotage et de réaliser des missions d'investigation et de surveillance auprès des forces armées, sous l'influence du N.K.V.D. d'Orlov, il ne tarda pas à pénétrer dans tous les secteurs de la zone républicaine (15).

Un des procédés utilisés pour tisser la légende noire du S.I.M. est l'amalgame : il mélange le fait qu'une partie des camps de concentration créés par le ministre anarchiste Juan García Oliver le 26 décembre 1936 (16) étaient passés sous l'administration du S.I.M. à un autre fait non précisément daté ni rigoureusement établi : la pratique de la torture dans les fameuses "checas ", par exemple dans la prison installée à Valence dans l'ancien couvent de Santa Ursula ou à Barcelone la prison de la rue Puerta de Angel. Ces lieux dépendirent longtemps du D.E.D.I.D.E., et ensuite seulement du S.I.M. Or la période pendant laquelle cette prison de Santa Ursula accueille de nombreux antifascistes victimes de l'appétit d'épuration du P.C.E. est antérieure au moment où elle passe sous le contrôle du S.I.M., avec tout l'appareil du D.E.D.I.D.E. dont elle dépend (17).

Mais si l'on cherche le " complot " ou si l'on dénonce le " contrôle communiste", peu importe la concordance des dates et l'absence de toute preuve documentaire:

plet sur la vie politique républicaine pendant la guerre civile. C'est une œeuvre gigantesque, celle de toute une vie, indispensable à qui veut approfondir l'étude de ce conflit et s'orienter dans l'immense bibliographie existante. On pourra lire avec profit un bilan des défauts et des qualités de l'ouvrage dans J. ARósTEGUI SÁNCHEZ, "Burnet Bolloten y la guerra civil española : la persistencia del "Gran Engaño" ", Historia Contemporánea, 1990, p. 151-177.

(14) B. Bolloten, La guerra civil española..., op. cit., p. 219-220.

(15) Ibid., p. 897. L'auteur ne fournit nulle part de démonstration à l'appui de cette thèse. Le chapitre sur le S.I.M. intitulé L'influence communiste dans les services de sécurité (p. 897-912) se contente de juxtaposer les citations de livres de mémoires et d'intercaler des affirmations péremptoires comme celle-ci : "Dans la majorité des cas, le S.I.M. préféra s'occuper de l'ennemi politique plutôt que de l'ennemi fasciste". Il cite l'ancien dirigeant du P.O.U.M., Gorkin, auteur d'une histoire de la persécution de son parti intitulé Un procès de Moscou en Espagne et d'un autre livre sur la politique stalinienne, Caníbales políticos (Méjico D.F., Quetzal, 1941) dans lequel on lit : "Ceux-ci [les staliniens] ont entre leurs mains le plus effrayant des appareils... Le S.I.M. arrête selon son caprice qui il veut arrêter... Le ministre de la Justice lui-même se sent impuissant face au S.I.M. Cet organisme fait peser la terreur sur les juges, les avocats, les procureurs" (p. 170-171).

(16) La création de ces camps avait pour but de désengorger les prisons et d'enfermer les prisonniers de guerre et les prisonniers d'opinion pro-franquistes.

(17) Que ces lieux de réclusion aient été à ce moment-là à mi-chemin entre la prison " privée" du P.C.E. et la prison officielle, ou qu'une partie de la police poursuivait des objectifs politiques particuliers, cela pose des questions passionnantes mais qui n'entrent pas dans le cadre d'une étude sur le S.I.M. lui-même. 
l'essentiel est d'affirmer la relation entre la répression stalinienne des opposants à Moscou et le S.I.M. Ensuite, il n'est pas difficile de faire fonctionner ce lien dans les deux sens : non seulement les actes extralégaux commis par le S.I.M. seront attribués à la vindicte tchékiste du P.C.E., mais aussi toute persécution des anarchistes et des poumistes pourra être mise sous le signe du S.I.M. Le responsable du P.O.U.M. Julián Gorkin, entre son arrestation en juin 1937 et son jugement avec les autres dirigeants de son parti, n'a à aucun moment dépendu du S.I.M., mais bien de la D.G.S., de la police, du D.E.D.I.D.E. et des attentions sinistres d'Orlov.

La "démonstration " de Bolloten rencontre un autre problème, le fait que Prieto rapporte dans ses mémoires sa lutte contre les communistes pour les empêcher de prendre le contrôle du S.I.M. Puisque le P.C.E. réclamait au moins un commandement régional du S.I.M., il nomma d'abord responsable pour la région de Madrid le communiste Gustavo Durán. Mais Durán engagea immédiatement 400 agents, tous communistes, sans prévenir le ministre. Au terme d'un affrontement direct avec Orlov et tout l'état-major cominternien en Espagne, Prieto réussit à imposer sa destitution au bénéfice d'un homme à lui, vieux militant socialiste, Angel Pedrero, qui révoqua toutes les nominations de son prédécesseur et n'engagea presque que des socialistes. De même, à la direction générale du S.I.M., Prieto nomma en premier son ami Angel Díaz Baza, qui, incompétent, fut remplacé rapidement par le républicain Prudencio Sayagües, et tout aussi rapidement par le socialiste Manuel Uribarri, lequel fuyait hors d'Espagne en avril. Le dernier chef du S.I.M. fut le socialiste proche de Juan Negrín, le président du Conseil, Santiago Garcès Arroyo, à son poste jusqu'à la fin du conflit.

La bataille pour le "contrôle politique " du S.I.M. avait donc apparemment été gagnée par le Parti socialiste ouvrier espagnol (P.S.O.E.). Mais pour la légende noire, il ne s'agit que d'une apparence trompeuse. On voit souvent apparaitre le thème du camouflage, le thème des "taupes" dans le parti socialiste : tous ces dirigeants, formellement socialistes, auraient en réalité été discrètement " gagnés " par les communistes. Certains auteurs, plutôt que de parler de " taupes ", préfèrent, pour contourner la difficulté que représente l'absence de commandement du S.I.M. aux mains du P.C., faire intervenir des adjoints communistes tout puissants qui sont censés avoir pris l'ascendant sur les responsables en titre. Plusieurs des auteurs invoquent la faiblesse de caractère d'Uribarri, le responsable de l'appareil central du S.I.M. installé à Barcelone, et en font une marionnette aux mains des "conseillers soviétiques". Son successeur à partir d'avril 1938, le négriniste Santiago Garcès, est censé être sous l'influence du directeur de la sécurité de Barcelone, Méndez.

Même si l'on considère que ces affirmations sont vraisemblables, à cause notamment de la position de leurs auteurs (18), que peut-on en tirer de bien précis? Peut-on

(18) Pour Uribarri, c'est Prieto qui l'affirme (Convulsiones de España..., op. cit.) ; pour Garcès, on trouve cette opinion dans un rapport interne et secret du service de renseignements de la C.N.T. daté du 25 juillet 1938 (que Bolloten s'empresse de citer p. 229 de son livre). Quant au couple Commandant Sierra/Loreto Apellániz, on doit cette affirmation au socialiste J. MARTínEZ AmUTIO (Chantage a un pueblo, Madrid, G. del Toro, 1974) qui a été gouverneur civil de la province d'Alicante. C'est le seul qui donne des précisions sur la carrière d'Apellániz, qui finit par être mis à pied et jugé à la fin de l'année 1938. 
inférer à partir de là une soumission complète de l'appareil du S.I.M. au Parti communiste? On est aussi en droit de se demander ce que signifie de dire " qu'untel est un agent du Parti communiste". On verra plus loin que dans ce domaine, on ne saurait être trop prudent.

Le S.I.M. est la plupart du temps considéré comme un organisme fortement centralisé. Une relation d'équivalence est souvent faite entre l'appareil lui-même et l'homme ou la poignée d'hommes qui le dirigent. De même, de nombreux auteurs réduisent le S.I.M. à ses activités civiles et attribuent à tout le service des abus ou des caractéristiques qui sont rarement prouvées. Tous les thèmes tels que l'arbitraire, les brutalités, la torture, les prisons secrètes, l'épuration stalinienne, la toute puissance et l'impunité absolue se retrouvent réunis dans une nébuleuse appelée S.I.M., d'autant plus secrète et inquiétante qu'elle reste méconnue et dépourvue d'archives.

Avant de revenir plus précisément sur cette question d'un hypothétique contrôle camouflé du S.I.M. par le N.K.V.D., il faut décrire ce qu'il était précisément, quels étaient son recrutement, son organisation, ses activités et ses réalisations, autant qu'il est possible de le faire pour la région de Madrid grâce à l'examen des déclarations recueillies du procès Pedrero (19). Cette description de l'organigramme et de la vie du S.I.M. est une synthèse que nous avons opérée en confrontant les interrogatoires de plusieurs dizaines d'agents et de responsables du S.I.M., les dépositions d'une quinzaine de ses "victimes " et les quelques pièces d'archives de l'organisme qui ont échappé à la destruction. Tous ces documents se trouvant dans le procès instruit contre Pedrero et consorts par les juges de la Causa General. Nous n'avons retenu que les éléments que l'on retrouve dans au moins plusieurs déclarations de prisonniers isolés les uns des autres. Pour cette première description du S.I.M. tel qu'il fonctionnait réellement, nous avons tenté d'échapper le plus souvent possible à la distorsion introduite par les tentatives faites par chacun pour minimiser son rôle personnel dans tel ou tel domaine d'intervention, ce qui donne peut-être une impression de schématisme que nous regrettons.

(19) Sauf indication contraire, toutes les informations renvoient aux cartons 1531 et 1532 de ce fonds. Les juges instructeurs du procès cherchent par leurs questions à éclairer un certain nombre d'aspects qui ne nous intéressent pas forcément. Des thèmes reviennent de façon obsessionnelle dans les interrogatoires, comme les banquets organisés par Pedrero dans un Madrid souffrant de la faim et du rationnement, la question de savoir si c'était bien une des maitresses de cet immoral chef du S.I.M. qui commandait en dernière instance. D'autres nous intéressent plus comme les relations avec les Russes ou la question des mauvais traitements infligés aux prisonniers. D'une façon générale, les enquêteurs du procès cherchent à reconstituer d'abord un organigramme général du service, avec tous les noms et le passé politique de chacun (et ils font appel pour cela à toute la documentation qu'ils peuvent rassembler au-delà de la Causa General, en incluant les fichiers de police d'avant la guerre), avant de déterminer l'intervention précise de chacun dans la réalisation de tel ou tel " méfait " précis, que ce soit l'arrestation, le meurtre, le tabassage d'un individu ou la participation à un coup de filet dans le milieu de la cinquième colonne. L'organisation générale du S.I.M. et les missions de chaque bureau, telles que Pedrero les restitue, sont confirmées par la responsable du bureau du personnel, agent franquiste, à qui les juges demandent en plus de reconnaître les photos de tous les employés et de confirmer ce qu'ils savent de leurs affectations. 

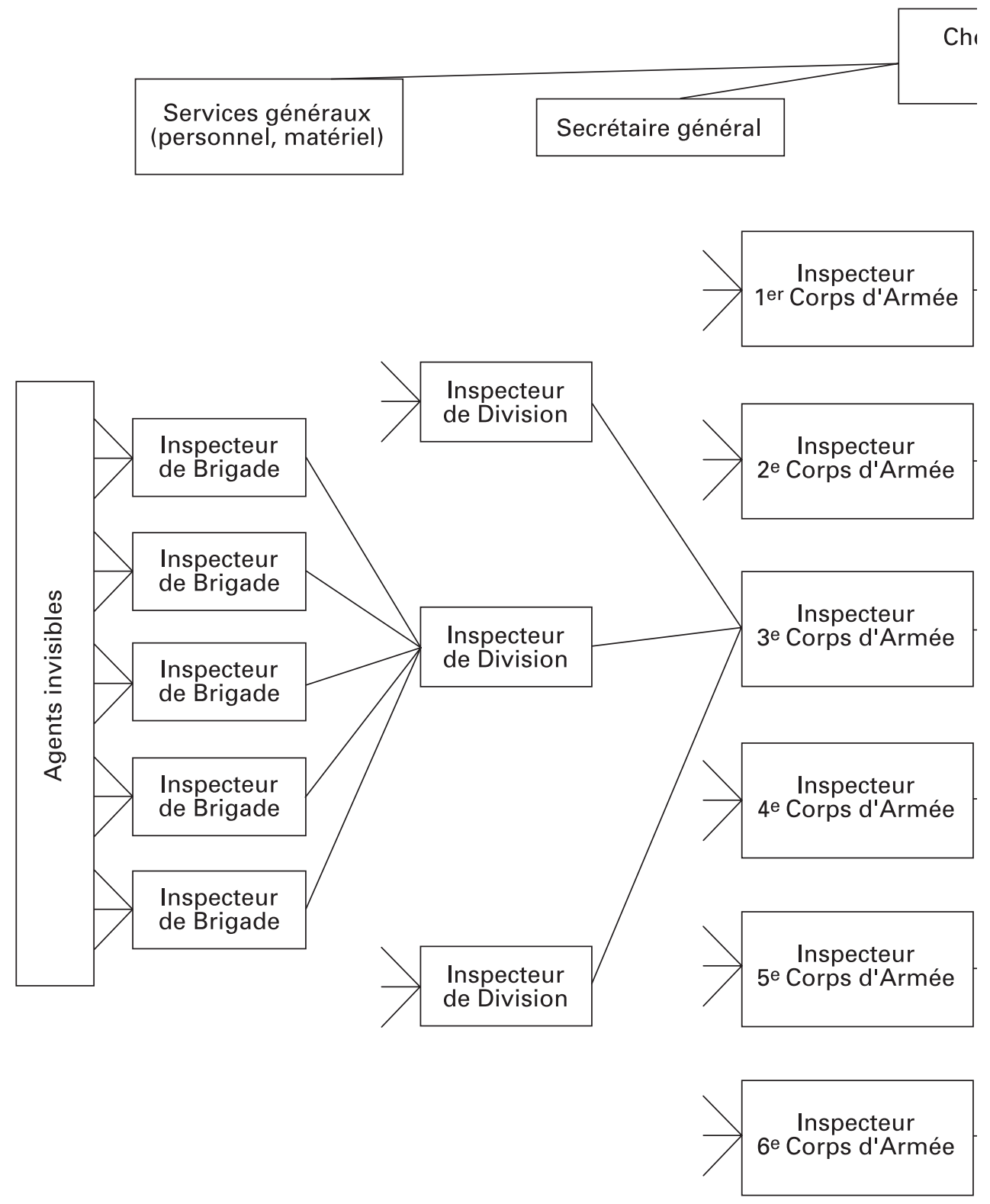


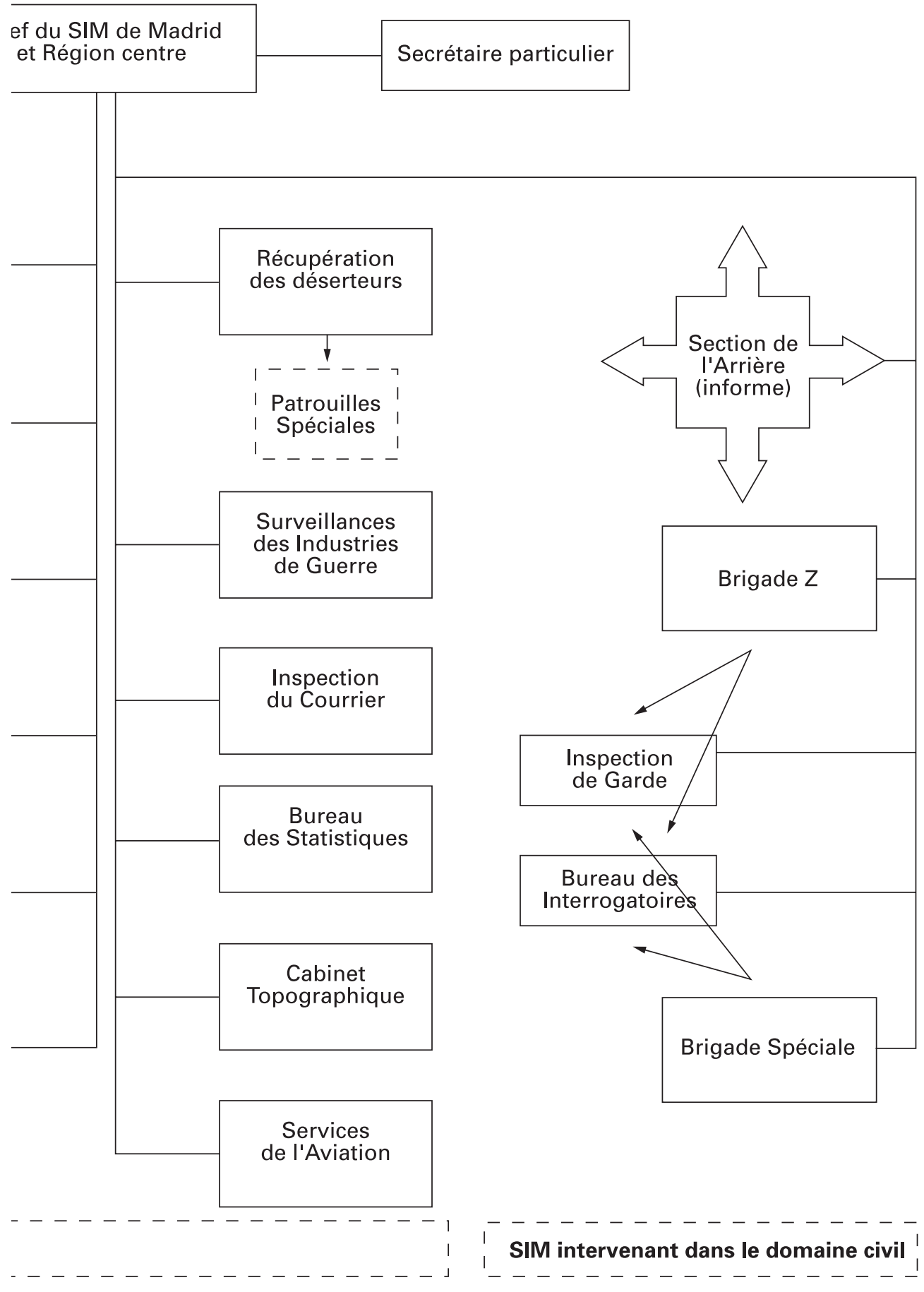




\section{Le S.I.M. de l'armée du centre : description d'un organe d'Etat en guerre}

Après l'éviction de son prédécesseur, le communiste García Durán, l'organisation du S.I.M. de l'armée du centre fut le fait propre d'Angel Pedrero García. A l'intérieur de chaque région militaire, les responsables qui ont les premiers mis en place et dirigé le S.I.M. avaient une certaine autonomie dans l'organisation concrète des services, même si les missions et le fonctionnement général se ressemblaient beaucoup (20). Il existait aussi des normes nationales, par exemple sur l'organisation des délégations au front, la prohibition de l'exécution des fuyards et des espions par les chefs d'unité, le monopole des détentions militaires par le S.I.M. ou la prohibition de circulation nocturne des voitures sans autorisation de l'état-major.

Pedrero, un juriste, était membre du P.S.O.E. depuis 1930, d'abord à Cáceres, en Estrémadure, où il organisait des écoles du soir et tentait d'attirer les intellectuels et les magistrats au Parti socialiste. Il avait aussi été délégué de la Fédération Provinciale des Travailleurs de la Terre du syndicat socialiste Unión General de Trabajadores (U.G.T.). Il se situait dans le P.S.O.E. dans l'aile droite, laquelle avait Julián Besteiro pour chef de file. Au début du conflit il avait fait partie des premières brigades de police, notamment de celle qui fonctionnait sous la direction de García Atadell (qui reçut plus tard des franquistes une réputation sanguinaire) (21). En décembre 1936, il fut chargé par Manuel Salgado, un des responsables militaires de Madrid et un des dirigeants locaux de la Confederación Nacional del Trabajo (C.N.T.), la centrale anarcho-syndicaliste, de constituer une petite brigade spéciale dont la mission devait être de surveiller les personnes qui fréquentaient le ministère de la Guerre. Il appelle alors neuf hommes, des cadres comme lui du Parti socialiste, déjà expérimentés dans le travail policier ou ayant collaboré avec lui dans la brigade de

(20) Il nous est malheureusement impossible de comparer avec suffisamment de précision nos connaissances du S.I.M. de Madrid avec les autres régions, pour lesquelles nous nous appuyons essentiellement sur les polémiques entre les partis sur l'activité du S.I.M. dans l'armée et sur les dossiers d'instruction transmis par le S.I.M. aux tribunaux d'espionnage, notamment en Catalogne. Seul D. PASTOR PETIT (La cinquena columna a Catalunya, op. cit., p. 222-223) propose une sorte d'organigramme du S.I.M. central qui comprendrait 13 sections : relations extérieures, aviation, armée de terre, marine, génie, armement, questions économiques, justice, transports et communications, instruction publique et spectacles, partis politiques et organisations syndicales, auxquelles il faudrait ajouter une trésorerie, et les départements d'intendance, de transports, de matériel et les services juridiques. Il décrit ensuite l'organisation de chacune des sections en huit bureaux. Outre que l'organigramme parait trop simple et que l'auteur ne cite pas sa source, cette structuration est en contradiction avec certains faits qu'on ne détaillera pas ici. On peut juste remarquer que la relation avec les différentes agences régionales n'apparait nulle part.

(21) A l'automne 1936, au moment où Madrid menace de tomber dans les mains de Franco et au plus fort de la résistance, la répression des secteurs nationalistes et de tous ceux qui paraissent soutenir les militaires est assez expéditive. García Atadell, qui y a participé, a la mauvaise idée de vouloir fuir l'Espagne les poches pleines de bijoux volés à ses victimes et est pris par les franquistes en décembre 1936. Ils organisent alors un procès aux forts accents de propagande : cet homme doit devenir la preuve de la fureur sanguinaire des "rouges". Sa mauvaise réputation vient en grande partie de cet épisode et du fait que sa fuite et son appétit de lucre lui ont attiré peu de défenseurs républicains. 
García Atadell : des hommes sélectionnés pour leur compétence et leur proximité politique.

Ce sont les mêmes neuf hommes qu'il recrute et place à la tête des principaux bureaux et départements quand Prieto le charge d'organiser le S.I.M. dans la démarcation de l'armée du centre (qui couvrait Madrid et ses environs). Deux d'entre eux, Octaviano Souza et Baltasar Fernández, deviennent respectivement secrétaire général du S.I.M. et secrétaire particulier de Pedrero. Pour chacun des corps d'armée (il $y$ en eut jusqu'à six), il nomme inspecteur un autre de ses collaborateurs proches. Chacun recrute ensuite à chaque échelon en fonction des mêmes critères de proximité politique et de compétence technique, ce qui donne la majorité au Parti socialiste au sein du S.I.M. Les inspecteurs de corps d'armée nomment à leur tour des inspecteurs dans chaque division et dans chaque brigade. Les inspecteurs de brigades nomment des " agents invisibles " connus d'eux seuls. Le critère de recrutement est autant la connaissance personnelle des futurs collaborateurs que l'appartenance à un même parti, qui joue comme une condition de la confiance plus que comme une raison idéologique.

Selon la taille des corps d'armée, on avait alors entre 15 et 30 inspecteurs du S.I.M., le nombre d'agents étant beaucoup plus important. Le personnel comptait en tout 1200 individus, dont les deux tiers pour la partie militaire de la mission du S.I.M. et le tiers pour l'organisation générale et les missions de police dans la ville (voir plus loin). Il faut ajouter un nombre d' "agents invisibles " dans les différentes unités militaires que l'on peut estimer à 4000 . Ces agents n'étaient pas des policiers du S.I.M., pourvus d'une carte, rétribués et autorisés à pratiquer des arrestations. Ils étaient de simples informateurs chargés de surveiller l'état d'esprit de la troupe et de repérer les candidats à la désertion, au passage à l'ennemi ou les membres de la cinquième colonne et les provocateurs. Selon Pedrero, "leurs avantages consistaient en des permissions ou autres bénéfices mineurs".

Une fois les suspects repérés, ils étaient arrêtés par les agents véritables et jugés par les tribunaux militaires, qui fonctionnaient régulièrement, à l'image des autres juridictions de ce type dans d'autres armées régulières (22). Les missions du S.I.M. dans l'armée ne s'arrêtaient pas là. Il gérait aussi la censure militaire : le chef de la centrale de Courrier en Campagne de l'Armée du Centre envoyait au S.I.M. les extraits des lettres détectées, repérées grâce au travail de ses deux mille agents censeurs. La correspondance civile était surveillée de la même façon, la centrale de censure envoyant au S.I.M. et à la D.G.S. toutes les lettres suspectes. La correspondance avec l'étranger était du ressort direct du chef de ce bureau, qui avait une équipe de traducteurs sous ses ordres.

On retiendra une autre mission importante qui était la "récupération des déserteurs". Ce service envoyait des imprimés au domicile de ceux qui ne s'étaient pas

(22) Sans que nous ayons pratiqué longtemps les archives des Tribunaux Militaires de l'Armée du Centre, le travail à partir d'un certain nombre de documents, notamment des demandes de renseignements et les fiches individuelles des soldats accusés adressées à leurs supérieurs ou les fiches de conclusions provisoires de l'accusation, suffit à nous convaincre que ces tribunaux n'étaient pas les machines à purges politiques que certains récits permettent d'imaginer. 
présentés spontanément à l'appel de leur classe. Au besoin, des patrouilles spéciales de soldats, commandées par des officiers du S.I.M., étaient chargées de les arrêter, de les interroger et de les renvoyer aux centres de recrutement ou dans des bataillons disciplinaires. Le S.I.M. contrôlait aussi la production des industries de guerre et avait des agents visibles et des agents invisibles dans les grandes usines pour repérer d'éventuels saboteurs. Il s'occupait même d'élaborer dans son bureau de statistiques des pourcentages sur les veuves de guerre ou sur la contribution en termes d'hommes des diverses capitales, Madrid, Barcelone ou Valence. D'autres services apparaissent dans l'organigramme comme le Cabinet topographique ou le service d'aviation, ainsi que les détachements pour certaines zones militaires, mais la source ne nous donne aucun détail car les juges franquistes ne s'y intéressent pas (23).

L'emprise du S.I.M. sur l'armée était forte et il y était sans doute omniprésent comme le colporte la légende. Pourtant la simple énumération de ses différents bureaux permet de mesurer la régularité d'un organisme que sa mauvaise réputation réduit à ses trop fameux abus. Ceux-ci existaient sans doute, comme le laissent penser les dénonciations de Manuel de Irujo. Cette description systématique nous présente une image du S.I.M. peu habituelle, celle d'une bureaucratie consciencieuse qui effectue un travail régulier ressemblant fort à ce qu'on trouve dans d'autres armées. Nous sommes loin de la police politique cruelle évoquée plus haut. La multiplicité de ses missions en fait certes un organisme impressionnant, mais on peut tenter de l'expliquer d'une autre façon que par l'appétit de puissance ou la recherche d'un contrôle politique total de la société.

Alors que sa légende noire nous le présente comme la police politique de l'arrière, le S.I.M. est avant tout lié à l'armée. Il naît à un moment important de la vie politique de la République en guerre, à la fin de l'été 1937, après que les partisans de la légitimité et de l'État républicain ont remporté une victoire politique définitive. Une fois le gouvernement de Largo Caballero tombé, une fois matée la volonté révolutionnaire des anarchistes à Barcelone lors des affrontements de début mai 1937 (24), la centralisation de l'État dans son entier se renforce et il regagne complètement des prérogatives qui avaient cessé un moment d'être les siennes.

L'Armée populaire de la République, avec la conscription obligatoire de plu-

(23) De même, s'ils cherchent à connaître les différents bureaux de l'administration interne du service, chargés de la tenue des dossiers personnels, du paiement des notes de frais et des agents du service de presse intérieure (qui éditait un Bulletin bi-hebdomadaire d'information du S.I.M. pour les agents) ou des conseils juridiques (chargés de vérifier la conformité des dossiers d'instruction transmis aux tribunaux), c'est essentiellement pour déterminer avec précision les responsabilités de chacun des individus arrêtés par les armées de Franco.

(24) Ces affrontements qui eurent lieu entre le 3 et le 6 mai 1937 à Barcelone et qui touchèrent aussi largement la Catalogne firent plusieurs centaines de morts. Ils virent s'affronter les ouvriers de Barcelone, majoritairement anarchistes, qui crurent à un nouveau soulèvement militaire, et les forces de sécurité, accompagnées et sans doute mues par la volonté du Parti Socialiste Unifié de Catalogne (P.S.U.C.), parti adhérent à la III Internationale et dirigé par le cominternien hongrois Gerö, de provoquer une confrontation décisive. Cette crise provoqua directement la chute du gouvernement Largo Caballero, la persécution du P.O.U.M., la fin de la participation de la C.N.T. au gouvernement (pour un an), la perte du contrôle de la police par la Catalogne et une vague d'arrestations importante dans les milieux révolutionnaires. 
sieurs classes, tend à devenir une "armée comme les autres", pourvue de services centraux et d'un état-major qui fonctionnent régulièrement. Il n'est plus question de se battre "contre le fascisme et pour la révolution ", mais "contre le fascisme et pour la République". D'autres problèmes subsistent pourtant et ils ne sont pas négligeables. La nouvelle armée populaire compte toujours dans ses rangs de nombreux militaires de carrière, toujours suspects. L'afflux de jeunes mobilisés par la conscription et non plus par le volontariat politique entraîne d'autres problèmes d'indiscipline que ceux qui existaient déjà, à un moment où la guerre déjà installée dans la durée offre des perspectives de plus en plus sombres. A cela s'ajoutent les problèmes de ravitaillement qui contribuent à créer le mécontentement chez ceux qui, dans les tranchées ou à l'arrière, ont de moins en moins de raisons d'être convaincus.

La création d'un organisme comme le S.I.M., qui est à la fois une police militaire centralisée et un service de contre-espionnage, répond à toutes ces transformations et doit être replacée dans le mouvement général de centralisation du commandement et de recréation des organes militaires "classiques" comme les tribunaux militaires. L'histoire du S.I.M., c'est aussi l'histoire de l'État qui se reconstruit et de sa nature. N'oublions pas non plus qu'au fur et à mesure que les revers s'accumulent, l'ombre de la "cinquième colonne " et des saboteurs, qu'il s'agit de pourchasser et d'éliminer, grandit aux yeux des antifascistes. Cette tâche est largement acceptée et nombreux sont ceux qui sont prêts à y contribuer, ce qui rend relativement crédible la déclaration de Pedrero sur l'enthousiasme des "agents invisibles" dans les unités militaires.

\section{Le S.I.M., témoin de la militarisation de l'arrière et des faiblesses de l'Etat}

Le S.I.M. étant avant tout un organisme militaire, il n'intervient de manière importante dans les villes et auprès des civils qu'à partir du printemps 1938, quand le D.E.D.I.D.E. est dissous et certaines de ses équipes sont intégrées au nouveau S.I.M. La plupart de ses interventions consistent en la poursuite des réfractaires au service militaire. Pourtant c'est cet aspect qui intéresse en premier lieu les juges franquistes : la majorité des interrogatoires détaillent le fonctionnement et l'action des services du S.I.M. qui opèrent à Madrid parmi la population civile. En termes de personnel, c'est la partie la moins importante du S.I.M. Cette fusion des deux services tient à la réputation d'inefficacité générale du D.E.D.I.D.E. et au fait que les missions se chevauchaient puisqu'ils étaient tous les deux censés faire du contre-espionnage.

Pour comprendre cette extension à un terrain d'intervention " civil ", il faut replacer cette croissance du S.I.M. dans le contexte d'accroissement des tensions politiques à partir d'avril-mai 1938. Le deuxième gouvernement Negrín nait le 5 avril 1938 après l'éviction d'Indalecio Prieto de son poste de ministre de la Défense pour défaitisme. Negrín, qui était déjà Président du Conseil, devient en plus ministre de la Défense et donc chef suprême du S.I.M. La campagne contre Prieto avait été 
orchestrée par le Parti communiste, mais aussi largement facilitée par les protestations de la C.N.T. contre le défaitisme ambiant et sans doute par un certain affaiblissement de la position du ministre au sein de son propre parti, le P.S.O.E. Le nouveau gouvernement Negrín s'oriente clairement vers une sorte de militarisation de la société, en particulier avec les projets de décrets de militarisation des industries de guerre et des tribunaux spéciaux qui depuis l'été 1937 connaissaient les causes d'espionnage, haute trahison et défaitisme et dont le fonctionnement était déjà passablement arbitraire (25). L'extension du domaine d'intervention du S.I.M., lequel n'a pas le monopole de l'arbitraire, correspond à ce mouvement général.

Au terme de la réorganisation qui a suivi l'absorption du D.E.D.I.D.E., les services du S.I.M. à Madrid dans le domaine " civil " se présentaient de la façon suivante. Trois services spéciaux et deux lieux de réclusion étaient assistés par deux petits bureaux, celui des interrogatoires et l'Inspection de Garde, installés dans le bâtiment du ministère de la Marine. Ces deux bureaux s'occupaient de l'accueil des détenus, de la confection des fiches individuelles, transmises ensuite à la section d'information (laquelle constituait des dossiers et les transmettait à son tour à d'autres destinataires), et de l'interrogatoire des détenus de moindre importance.

Le premier des services spéciaux, la Section de l'arrière, intervenait à la fois sur des questions militaires et des questions civiles. Il était dirigé par Jesus Luque, vieux militant socialiste entré dans la police au début de la guerre sous les ordres de García Atadell, puis affecté aux Services Spéciaux du ministère de la Guerre. Il informait les autorités militaires sur les personnels qui venaient de l'arrière et surveillait les permissionnaires et les scandales qu'ils pouvaient produire à Madrid, notamment dans les spectacles publics et les débits de boissons. Il s'occupait aussi de la surveillance nocturne de la ville grâce à des unités spéciales de soldats et du transfert des prisonniers vers les bataillons de fortifications. Il devait en outre contrôler le personnel du génie, en particulier les ingénieurs, cadres non ouvriers dont l'origine sociale suscitait la méfiance. Il fournissait aussi une aide ponctuelle aux deux autres services spéciaux, la Brigade Spéciale et la Brigade Z.

Cette dernière, au nom inquiétant, était en fait chargée de réprimer le marché noir, qu'on soupçonnait d'être organisé ou du moins encouragé et entretenu par la fameuse cinquième colonne. Ce mystérieux ennemi dont le nom a fait fortune signifiait aussi bien les agents des services d'espionnage franquistes, les réseaux phalan-

(25) Il faut signaler en particulier le décret du 2 mai 1938, qui étend la compétence des Tribunaux Spéciaux de Garde (Tribunales Especiales de Guardia-T.E.G.) créés par décret du 29 novembre 1937 à tous les crimes d'espionnage, de défaitisme et de haute trahison et non plus seulement aux cas de flagrant délit. Ces crimes ont été définis dans le décret du 22 août 1937 qui crée le Tribunal Spécial d'Espionnage et de Haute Trahison (Tribunal Especial de Espionaje y Alta Traición). Le 2 mai, celui-ci devient tribunal d'appel pour les T.E.G., du moins en théorie, car dans la pratique il était difficile de faire appel. On peut donner une idée de l'arbitraire des T.E.G. en indiquant qu'ils instruisaient souvent en moins de 24 heures et qu'ils jugeaient en moins de deux heures (d'où leur surnom de "photomaton "). La défense n'avait connaissance du maigre dossier que quelques heures avant le jugement. Les sentences les plus courantes étaient la mort (la plupart du temps commuée sur décision du conseil des ministres) ou des peines de réclusion de 30 et 20 ans. Les jurys étaient composés de trois représentants, un pour chaque ministère: Justice, Intérieur, Défense. 
gistes de saboteurs ou du Secours Blanc que les propagateurs de fausses nouvelles ou les personnes convaincues de propos défaitistes ou hostiles à la République. La vente à des prix prohibés, la constitution de stocks, la spéculation ou le trafic de fausse monnaie étaient devenus autant de délits intentionnels de sabotage qui entraient dans la sphère de compétence du S.I.M. Cette Brigade Z était dirigée par Fernando Valentí Fernández, dont on reparlera un peu plus loin.

La dernière Brigade, dirigée par Emilio Perailes Sauquillo, était directement chargée du contre-espionnage, de la découverte des réseaux franquistes. Ses réalisations étaient connues à l'époque par la publicité donnée à certaines affaires, publicité par laquelle le S.I.M. cherchait à se fabriquer une réputation d'efficacité et à se présenter comme indispensable. Les "affaires" découvertes étaient, comme tous les moindres reculs franquistes sur la ligne de front, autant de victoires précaires qu'il s'agissait de célébrer pour maintenir le moral. Pour l'occasion, on n'hésitait pas à faire passer un groupe de personnes arrêtées lors d'un spectaculaire coup de filet pour un réseau complet de la cinquième colonne qu'on aurait démantelé, ainsi des fameux " 195 " ou des " 165 " (26). Perailes explique à ses juges qu'on a beaucoup parlé de l'affaire des 195 à cause des soixante condamnations à mort requises malgré le peu d'importance des faits (vingt et une peines de mort ont été prononcées mais aucune exécutée) (27).

D'autres "réseaux" furent démantelés, comme un réseau de transmission de plans de fortifications républicaines, un réseau d'espionnage des communications téléphoniques, un groupe qui faisait circuler des affichettes de propagande défaitiste et des tampons et papiers à en-tête d'organismes officiels. Mais la plupart de ces affaires, en particulier celle des " 195 ", ne sont pas toutes à porter au crédit du S.I.M., du moins pas entièrement. La Brigade spéciale existait en effet sous une autre appellation au sein du D.E.D.I.D.E. et avait été reconstituée presque entièrement dans le S.I.M. De plus, elle ne travaillait pas seule : elle fut par exemple chargée d'interroger les inculpés dans l'affaire dite des "syndicats verticaux" qui avait été découverte par la police de Madrid. Cette collaboration avec les autres services semble beaucoup plus fréquente que ne le laisse accroire la légende noire qui a tendance à isoler le S.I.M. dans sa toute puissance opaque et inquiétante, comme un organisme supérieur sans lien avec l'appareil d'ordre public de l'État.

D'une certaine façon, notre source, la Causa General, a, un peu comme les livres cités plus haut, le défaut d'attribuer au S.I.M. la paternité d'enquêtes dont il n'est pas l'auteur. Sa grande visibilité et le fait que nombre de ses agents avaient travaillé dans d'autres services de police qui ont été ensuite absorbés ou recomposés fait que l'ensemble de leur comportement pendant la période de la guerre est consi-

(26) Une thèse récente, succombant à l'illusion documentaire, a même accrédité l'idée que ces groupes d'espions ou de membres de la cinquième colonne formaient autant de réseaux décrits et étudiés au même titre que l'autres organisations secrètes, ignorées du S.I.M., mais qui existaient bel et bien. J. CERVERA, Madrid en Guerra. La ciudad clandestina (1936-1939), Madrid, Alianza, 1998.

(27) Les réalisations du S.I.M. de Barcelone, pour autant qu'on puisse en juger par un examen rapide des dossiers judiciaires, semblent moins devoir à ce souci de publicité et atteindre plus directement ou efficacement les réseaux de la Phalange clandestine (cf. aussi D. PASTOR PETIT, La cinquena columna a Catalunya, op. cit.). 
déré comme celui d'agents du S.I.M. Là encore l'amalgame est facile à faire. Les "nombreux " abus dénoncés par Manuel de Irujo lors de sa démission sont à replacer dans l'histoire de l'appareil répressif de l'Espagne contemporaine : la difficulté éprouvée par l'État pour surveiller et contrôler ses propres organes de répression, la quasiabsence d'un appareil de police civil, professionnalisé et compétent, la militarisation continue de l'ordre public depuis des décennies, sont autant de tendances lourdes que l'on retrouve dans le S.I.M. Celui-ci, confondu avec les autres services répressifs de la République en guerre, concentre beaucoup de défauts et de faiblesses de l'État espagnol d'alors. Ce sont les deux questions sensibles de la torture et des liens avec les services soviétiques qui voient souvent la prudence de l'analyse le céder à la facilité de l'amalgame.

\section{Comment fonctionne l'amalgame : l'agent infiltré, la torture, et le contrôle par le N.K.V.D.}

Quand il est question de torture dans la bibliographie sur la guerre civile, c'est souvent de prisons secrètes ou clandestines - les fameuses "checas" - qu'il s'agit. Le S.I.M. de la région centre gérait en propre deux lieux de réclusion qui avaient appartenu au D.E.D.I.D.E. Le premier était la prison de San Lorenzo, installée dans un ancien collège religieux et d'une capacité d'accueil approximative de 200 prisonniers. Le second était le Camp de travail $n^{\circ} 1$, d'abord situé à Ambite, puis transféré à Cuenca. Lors de l'instruction du procès Pedrero, la question des mauvais traitements tient une large place dans les préoccupations des juges : ils interrogent une dizaine de témoins qui se disent victimes de mauvais traitements dans la prison de San Lorenzo. Les témoignages sont tous concordants pour dénoncer des passages à tabac fréquents et l'utilisation du froid et de la faim pour faire signer des aveux "préparés à l'avance" : deux cellules sont décrites, surnommées "la glacière " et "le réfrigérateur ", dans lesquelles les prisonniers étaient soit laissés nus dans de l'eau jusqu'aux chevilles, soit aspergés d'eau glacée pendant les mois d'hiver. Dans un livre franquiste de Regina García (28), on trouve la description des mêmes tourments, ce qui semble confirmer leur véracité. Pourtant, dans le procès-verbal d'une visite de reconstitution des juges de la Causa General, les principales victimes de mauvais traitements qui les accompagnent font des déclarations plus prudentes qui permettent de relativiser le contenu de leurs premières dépositions.

Les agents et les services mis en cause étaient la brigade de Perailes et celle de Valentí, ce qui n'a rien d'étonnant car c'étaient ceux qui pratiquaient le plus d'interrogatoires et étaient les plus importants pourvoyeurs en prisonniers. Mais à propos de Valentí, il est écrit qu'il avait la réputation d'avoir déjà infligé des mauvais traitements et d'avoir été sanctionné pour cela. En fait, Valentí représente une sorte de

(28) Yo he sido marxista. El cómo y el por qué de una conversión, Madrid, Ed. Nacional, 1952. 
cas type de relations ambiguës avec le Parti communiste, relations qui pourraient donner lieu à la constitution d'un très bel amalgame mêlant la torture, le P.C., le N.K.V.D., la chasse aux opposants, etc. On va voir pourtant que rien dans la documentation n'autoriserait à extrapoler ainsi.

Une autre source tendrait à confirmer cette réputation de violence de Valentí et de ses hommes. Une bonne partie des membres de la Brigade $Z$ avaient déjà travaillé sous ses ordres à la Brigade Spéciale du Commissariat Général de Madrid du 108 de la rue Serrano, qui avait été dissoute à la fin du mois de février 1938 suite à une enquête pour mauvais traitements. L'instruction démarra en octobre 1937 et ne se termina qu'à l'automne 1938 : elle conclut à la vraisemblance des accusations de passages à tabac répétés formulées par de nombreux prisonniers, mais les responsables ne furent pas sanctionnés. Entre-temps, en avril 1938, Valentí avait rejoint le S.I.M. à un poste moins exposé, la répression du marché noir (29).

D'autres policiers, souvent identifiés comme communistes, peuvent être fortement soupçonnés d'avoir pratiqué la torture, mais dans la plupart des cas, il est difficile d'y faire apparaitre le sigle S.I.M., car il ne s'occupe de civils et ne reprend les attributions du D.E.D.I.D.E. qu'assez tard (30). Si des cas de mauvais traitements sont vraisemblables, ils concernent d'autres organismes, et en attribuer la paternité au S.I.M. serait risquer l'anachronisme. Il ne s'agit pas de prendre prétexte de l'absence de documentation et de preuves pour rejeter a priori l'hypothèse de la pratique de la torture par le S.I.M. Il est en revanche important de retenir que si elle fut pratiquée, elle ne fut pas l'apanage de ce service, dont l'omniprésence à la fin de la guerre a effacé tous les autres (en particulier le D.E.D.I.D.E., mentionné par très peu d'historiens malgré sa responsabilité dans ce domaine).

Pour que l'amalgame sur lequel repose la légende noire du S.I.M. fonctionne correctement, il faudrait en outre établir le contrôle du S.I.M. par le N.K.V.D. Nous avons vu que Pedrero, responsable du S.I.M. de Madrid, avait été désigné par Prieto pour contrecarrer l'entreprise communiste de colonisation de ce service. Par la suite, cependant, s'instaure un modus videndi entre le chef du S.I.M. et les assesseurs soviétiques. D'après plusieurs dépositions, dont celle de Pedrero lui-même, il existait une collaboration active avec les hommes d'Orlov, malgré certaines périodes de froid, suivies par de nouveaux rapprochements. Pedrero insiste sur le fait que cette collaboration était avant tout technique, le S.I.M. de Madrid restant dominé par les socialistes.

(29) A.H.N., C.G., 1286.

(30) On dispose en outre d'un témoignage précieux sur ces questions pour Barcelone et Valence, celui de Pavel et Clara Thalmann (Combats pour la liberté, Paris, La Digitale, 1997) : ils furent incarcérés dans les prisons de Puerta del Angel et Santa Ursula comme trotskistes. Leur récit est exceptionnellement vraisemblable car certains détails sont confirmés par des rapports confidentiels de la D.G.S. au ministre de l'Intérieur (A.H.N., C.G., 614). Ils affirment avoir eu connaissance de tortures sur certains individus mais restent très prudents et notent le caractère exceptionnel et non systématique de ces pratiques. S'il est admis que le N.K.V.D. a torturé Andrés Nin avant de l'exécuter, il serait cependant imprudent d'extrapoler sur cette question. Les agents d'Orlov auraient eu toutes les raisons de torturer Thalmann, dirigeant trotskiste suisse, pour lui arracher une confession propre à renforcer l'accusation dans le procès du P.O.U.M. ; pourtant Thalmann n'eut à souffrir aucun mauvais traitement. Tous ces faits datent de 1937. 
C'est à ce moment que l'on retrouve la question des "taupes", des "agents" du Parti communiste dans le Parti socialiste : l'appartenance d'un certain nombre de policiers au P.S.O.E. ne serait que pure question de forme, ces hommes étant en fait autant de pions du P.C.E. qui, " champion " du camouflage et de la dissimulation, cherchait à ne pas attirer l'attention sur sa progression dans l'appareil d'État. Les juges de la Causa General, dans la partie qui traite des relations du S.I.M. avec les Russes, fournissent à cette hypothèse une possible confirmation. Le personnage central de ce chapitre n'est autre en effet que Fernando Valentí Fernández, dont on vient de voir qu'il est fortement soupçonné de brutalité. Il apparaît dans la documentation retrouvée par la police franquiste et dans le témoignage de Pedrero comme un vieux militant de Izquierda Republicana, le parti républicain de gauche.

Introduit par Cazorla (Commissaire Général de Madrid de janvier à avril 1937) auprès des assesseurs techniques de la police madrilène, les Russes de l'hôtel Gaylors, Valentí était manifestement au centre de cette coopération technique: il était l'homme de confiance du Commissariat Général dans les relations avec les Russes de l'état-major du Comintern et, en même temps, le répondant de la police espagnole pour les Russes. Il connaissait si bien les dirigeants de l'appareil soviétique qu'il leur est arrivé de lui confier une mission de la plus haute importance (31) : il avait, avec le Cominternien surnommé José Ocampo (32), organisé la petite brigade de policiers qui étaient allés en juin 1937 à Barcelone arrêter le Comité Exécutif du P.O.U.M. et en particulier Andrés Nin et l'avaient ramené à Madrid. Il semble même qu'il ait participé de très près à la confection de la fameuse "preuve " contre le dirigeant du P.O.U.M., le plan millimétré de Madrid signé au dos à l'encre sympathique d'un mystérieux $\mathrm{N}$, plan qui appartenait à un réseau de la cinquième colonne appelé "réseau Golfín ", du nom de son dirigeant, l'ingénieur Golfín. Certains des membres de cette équipée témoignent, tous appartenaient à la Brigade Spéciale de Valentí, la Brigade du 108, rue Serrano, cette fameuse "checa " où les prisonniers étaient maltraités.

A quelques anachronismes près, Valentí pourrait figurer comme le concentré idéal de la légende noire : véritable " preuve par neuf " que le S.I.M. était un appendice des tortionnaires du N.K.V.D. en Espagne. Pour cela, il suffirait d'en faire un agent communiste infiltré dans le Parti républicain. Ce raisonnement pourrait être étendu à Pedrero, "formellement " socialiste, et finirait par jeter le soupçon sur le S.I.M. dans son entier, Valentí n'étant peut-être que "la partie immergée de l'iceberg " communiste, de l'appareil de répression pro-soviétique. Pourtant ce raisonnement se heurte à un problème majeur : en février 1939, Pedrero participe acti-

(31) Les principaux témoignages sont ceux de Valentí lui-même, de Rosell, son acolyte et futur secrétaire de la Brigade Z, de Tomás Durán Gonzalez, autre membre de la police, ayant appartenu, lui, au P.C.E., de Cazorla lui-même, d'agents de base de la Brigade spéciale, d'un agent franquiste arrêté, interrogé puis relâché et d'un confident de la police, utilisé par Valentí à l'époque des faits. On n'a retenu que les aspects sur lesquels tous ces témoignages concordent (folii 226 à 233 de A.H.N., C.G., 1532).

(32) Décrit par certains comme un Russe (par extension sûrement car il évoluait au Gaylors parmi les Russes), par d'autres comme un Argentin (il avait un fort accent de ce pays), serait-ce Codovila? Les témoignages concordent aussi pour dire qu'après cette affaire, on ne l'a plus revu à Madrid, et sans doute en Espagne. 
vement avec le dirigeant socialiste Besteiro au complot et au coup d'État du général Casado contre le gouvernement Negrín et la politique de résistance à tout prix soutenue par le P.C.E. Dans ce mouvement, Valentí ne fait pas défaut et il participe à la chasse aux communistes assumée entre autres par le S.I.M. Que dire d'un tel agent qui ne préviendrait pas ses supérieurs politiques et qui même leur donnerait la chasse?

Il n'est pas le seul en cause : tous ses hommes quil l'accompagnent de la Brigade Spéciale au S.I.M. et dans toutes ses missions devraient eux aussi être des communistes infiltrés. Or rien ne les distingue de tous les autres agents du S.I.M. dont on connait le passé militant : presque tous sont membres de l'U.G.T. et du Parti socialiste depuis plusieurs années. Avant d'être agents du S.I.M., ils étaient miliciens de l'arrière et policiers, proposés à ces postes par l'organisation socialiste. A aucun moment leurs parcours ne permettent d'accréditer l'idée de la constitution d'une brigade secrètement communiste par Valentí. Leur recrutement semble répondre, comme pour l'ensemble du S.I.M., à des critères de professionnalité et des liens personnels (33).

A l'inverse, le S.I.M. est absent d'un cas "typique" de persécution de militants trotskistes par des policiers staliniens. Il s'agit de l'arrestation, de l'interrogatoire et du procès monté contre le groupe des Bolcheviques-Léninistes et en particulier de leur chef Munis. Dans cette affaire, pour laquelle il y a de forts soupçons qu'il a été fait usage de pressions physiques violentes, c'est la Brigade d'Inspection Criminelle de Barcelone qui intervient, en la personne du commissaire Javier Méndez et du cadre communiste Julián Grimau (34). Le cas est typique dans la mesure où il s'agit pour les policiers de faire avouer aux accusés qu'ils préparaient des attentats contre le chef du gouvernement sur ordre direct d'un fantaisiste "comité parisien de la IVe Internationale". Cette affaire se déroule de mars 1938 à janvier 1939 et à aucun moment le S.I.M. ne joue un quelconque rôle, alors qu'il est censé être le bras armé du N.K.V.D. en Espagne.

Ces éléments qui invalident l'amalgame faisant du S.I.M. l'organisme de terreur stalinienne ne nous conduisent à aucune conclusion sensationnelle, mais nous permettent de faire quelques remarques de méthode qui nous semblent importantes : ni le Parti communiste d'Espagne ni les services soviétiques n'avaient un contrôle efficace et sûr de l'appareil répressif qu'ils furent amenés à utiliser dans leur chasse aux oppositionnels. Pendant la guerre civile, des individus ont pu prêter leur concours à la politique communiste sans qu'on puisse pour autant en faire des pions, des hommes contrôlés par le P.C.E. En faire des agents doubles, des "taupes", relèverait du roman d'espionnage ou du déterminisme (35). Cela signifierait supposer par avance

(33) Julián Gorkin, dirigeant du P.O.U.M. arrêté par ces mêmes hommes à Barcelone et transféré par eux à Valence, confirme que les deux policiers qui le gardaient dans la voiture étaient des militants du Parti socialiste, fort étonnés du rôle qu'on leur faisait jouer en arrêtant les chefs d'un parti ouvrier et effrayés de la surveillance qu'exerçaient sur eux les agents du N.K.V.D., en particulier le mystérieux Olano (El proceso de Moscou en Barcelona, op. cit., p. 116-119).

(34) A.H.N., C.G., 1706. Julián Grimau fut plus tard secrétaire personnel de Santiago Carrillo, le dirigeant du P.C.E., et un des derniers fusillés de la lutte antifranquiste.

(35) Le mot " agent " véhicule à lui seul tout une logique policière comme le souligne C. SERRANO, "La 
qu'un parti ou un quelconque sujet puisse exercer un contrôle absolu sur des individus ou des institutions.

Dans le cas qui nous occupe, il faut rappeler des paroles comme celles de Gabriel Morón, Directeur Général de la Sécurité à Madrid, "soupçonné " par Bolloten à un moment d'être lui aussi une "taupe" :

Pendant les premières années de la guerre civile espagnole, tout ce que j'ai vu du comportement communiste a été la conduite sur le front des communistes dans les unités que je commandais et qui du point de vue du courage et de la discipline était du niveau de militaires professionnels. En conséquence (une conséquence naturelle en période de guerre), mon attitude envers eux, comme celle de presque tous les chefs républicains à ce moment-là, a été amicale. Elle est devenue de plus en plus hostile quand j'ai appris les méthodes implacables et la duplicité des communistes, leurs tentatives de contrôler totalement toutes les situations, quand j'ai appris que leur loyauté allait à leur parti avant d'aller au gouvernement qu'ils disaient servir et que, finalement, les instructions que le parti recevait ne relevaient pas de l'idéalisme mais d'intérêts très concrets qui étaient loin d'être ceux du peuple espagnol (36).

Aussi loin que puisse avoir été la collaboration et l'engagement de tels hommes (parmi lesquels on peut sans doute compter Valentí et Pedrero) aux côtés du et même dans le P.C., il serait abusif de faire d'eux des pantins aux actes prévisibles. Supposer ainsi le contrôle effectif et total d'un individu par un parti reviendrait à créer un lien causal mécanique et permanent entre une identité figée par une étiquette politique et une série d'actions censées former un système. Une fois ce lien établi, on perdrait de vue la complexité des relations et des situations politiques, l'épaisseur des raisons de l'action et il ne resterait plus qu'à raconter un synopsis, une intrigue avec ses révélations, ses manipulations, ses complots et ses responsabilités. En outre, on peut, dans le cas de la guerre civile espagnole, émettre des doutes sur l'existence de buts politiques conscients, positifs et non contradictoires, de la part des communistes. Leurs tentatives de contrôle de certaines parties de l'appareil d'État sont avérées, mais il ne faut pas en déduire trop vite l'existence d'une stratégie claire de conquête du pouvoir qui servirait ensuite dans le raisonnement à donner un sens a priori aux traces de l'activité communistes repérées dans la documentation.

guerre d'Espagne et l'antifascisme en question ", in M. PAPY (coord.), Les Espagnols et la guerre civile, Pau, Atlantica, 1999, p. 327-333 : "Le mot est lâché : agent ; avec ce qu'il implique de subordination, bien sûr, mais aussi de vénalités possibles; en tout cas de soumission à des intérêts autres, secrets et inavouables. Fourbe et peut-être double, l'agent, par nature, masque ses intentions, dissimule ses motifs, n'agit jamais pour les raisons qu'il invoque, ses propos cachent toujours une ultima ratio qui est sa véritable raison d'être [...]" (p. 329).

(36) G. Morón, Política de ayer, política de mañana, op. cit. 


\section{Au-delà de la région centre, comment poser la question du contrôle du S.I.M.?}

Nous avons vu que dans le cas du S.I.M. de la région centre, la légende noire est largement fausse. On doit tenter de répondre à présent à la question du contrôle du S.I.M. par le P.C.E. pour les autres régions, même si l'on sait que la légende noire fonctionne là aussi à partir de concepts erronés. Dans chaque région, un homme incarne la soumission aux noirs desseins du P.C.E. ou, mieux, du Comintern. Dans la région de Valence, c'est un certain Loreto Apellániz (37). Pour l'Andalousie, la légende noire a aussi son homme, un certain E. Francés (38). Pour le S.I.M. central, c'est d'abord Manuel Uribarri et ensuite Santiago Garcès, tous deux socialistes, qui semblent avoir été les vecteurs (et les paravents) de la présence communiste dans le S.I.M. Chacun s'en défend dans ses mémoires (39) et accuse l'autre d'avoir été un pantin. Il n'est pas dans notre propos de mener une étude détaillée sur ces questions, dont le but serait de rétablir la mémoire de tels hommes. Il nous semble plus important d'insister sur le fait que, comme dans le cas de Madrid, l'attribution d'un rôle à un individu, fût-il haut placé, ne peut en aucun cas tenir lieu d'explication des particularités et des activités d'un service comme le S.I.M. Si l'on s'écarte de ce raisonnement, on peut entrevoir toute la complexité des rapports politiques, en particulier la fragilité des équilibres et l'indétermination des situations autour de "l'objet S.I.M. ".

Des rapports internes de la C.N.T. sur la question du S.I.M. nous offrent un aperçu de cette complexité. Ces documents datent de juin et juillet 1938, au moment où le P.C.E. est censé atteindre le comble de son contrôle sur le S.I.M. (40). Garcès demande à la C.N.T. de lui proposer un homme de confiance pour figurer aux côtés de l'assesseur russe du S.I.M. central, ce qui ne laisse pas de surprendre vu ce que sont censés être les rapports entre la centrale anarcho-syndicaliste et le S.I.M.

Le rapport de Iñigo, responsable de la C.N.T., expose les problèmes tels que Garcès les lui a confiés :

On impose au S.I.M., sous prétexte de formation extraordinaire et d'expérience, la présence d'un Russe (dont nous ne connaissons pas le nom) pour qu'il dirige ses tâches spécifiques. Dans le but de neutraliser la politique d'absorption que ces éléments développent partout, Garcès [...] veut placer à ses côtés une paire de collaborateurs qui

(37) Cf. note 18.

(38) J. MARTínez Amutio (Chantage a un pueblo, op. cit.) dit de lui qu'il était socialiste de tendance négriniste, mais qu'il était totalement acquis au P.C. et à sa politique.

(39) M. URIBARRI, La quinta columna española : revelaciones sensacionales. El S.I.M. de la Répública, La Havane, 1943. Pour Santiago Garcès, on peut lire une transcription d'une interview réalisée par D. PASTOR Petit, Los dossiers secretos de la Guerra Civil, op. cit., p. 115-119.

(40) Santiago Garcès est censé selon la légende noire du S.I.M. avoir offert plus de facilités aux Soviétiques que son prédécesseur Manuel Uribarri. Selon D. PASTOR PETTT (ibid.), celui-ci aurait fui l'Espagne en auril 1938, entre autres parce qu'il ne supportait plus les pressions des Russes, dont il avait fini par avoir peur car ils lui reprochaient de ne pas collaborer avec suffisamment de discipline. 


\begin{abstract}
limitent son totalitarisme et sa conception exclusive et personnelle des travaux à réaliser, de manière à affaiblir son influence. [...] Garcès nous a indiqué qu'il serait bon que, pour éviter les soupçons du Russe, lequel répugne à la collaboration de la C.N.T., notre camarade Campon entre dans le service avalisé par un parti républicain. Cela nous indique clairement que l'influence moscovite est si forte que Garcès est obligé à recourir à des trucs comme celui-ci pour la contourner (41).
\end{abstract}

La forte présence des Russes ne s'accompagne pas automatiquement d'un contrôle hégémonique : chaque organisation politique ruse avec les autres en fonction de rapports de force politiques qui ne s'établissent pas seulement au niveau du gouvernement, mais aussi dans les autres instances et organes de l'État et dans le poids des organisations politiques elles-mêmes (nombre de militants compétents et disponibles). Ces rapports de force évoluent de façon complexe et rapide tout au long du conflit : le S.I.M., là non plus, n'est pas le bras armé du N.K.V.D. chargé de la répression des opposants.

D'autres documents nous apprennent que cette période du printemps et de l'été 1938 voit se régulariser quelque peu les rapports entre le S.I.M. et la C.N.T. Un rapport interne de la section défense de la C.N.T. sur les abus du S.I.M. décrit un certain nombre d'actes arbitraires, mais nulle part il n'est question de persécution politique des anarchistes par les communistes. Quand la section juridique anarchiste fournit à Iñigo la liste des prisonniers qu'a l'organisation dans les prisons du S.I.M., ils ne sont qu'une cinquantaine; la plupart relèvent de problèmes de discipline et quelques-uns seulement de la lutte politique entre les anarchistes et leurs adversaires (qui ne sont pas seulement des communistes). Le S.I.M. n'apparait pas plus lié aux persécutions politiques d'une partie de l'anarchisme et des communistes d'opposition que d'autres services de sécurité. Les centaines de "prisonniers antifascistes " qui peuplent les deux premières galeries de la prison de Barcelone n'ont pas eu affaire à lui.

On aimerait pouvoir aller plus loin et faire une étude positive du S.I.M. Service de contre-espionnage et police militaire, il est avant tout une partie particulièrement visible, quoique opaque, de l'appareil d'État. Par ses fonctions, il est l'objet de toutes les légendes. Par sa nature, service de sécurité d'un État qui se reconstitue en pleine guerre civile, il est le terrain de luttes politiques fortes entre les organisations sur lesquelles s'appuie l'État. Le fonctionnement de sa légende noire est à l'image de bon nombre de récits de la guerre civile espagnole. Les difficultés qu'elle révèle pour envisager la politique passée autrement qu'en termes d'étiquettes, de caractérisation, de "lignes politiques", de contrôle et de manipulation nous renvoient aux difficultés propres de l'historien dans le champ de l'histoire politique du contemporain : articuler la complexité du réel politique, l'épaisseur des relations politiques, à la manière dont les acteurs eux-mêmes rendaient compte des situations et des conflits.

(41) Rapport du 26 juillet 1938, International Institute of Social History, C.N.T., Pq. 74 C2. 